\title{
UNASUR Y SUS POLIITICAS PARA LAS PYMES EN COLOMBIA: CASO BOGOTÁ
}

\author{
Fecha de recepción: 11-06-2009, aprobación: 10-09-2009
}

Ángela Julieta Mora Ramírez

\section{RESUMEN}

En este artículo se analizarán las actuales políticas de la UNASUR con las Pymes exportadoras. Desde la perspectiva de la integración regional se hará énfasis en el caso colombiano y específicamente en el bogotano.

\section{ABSTRACT}

This article develops an analysis of current policies of UNASUR to exporting SMEs, at present especially in Colombia in Bogota, from the vision of regional integration.

\section{PALABRAS CLAVE}

Pymes exportadoras, integración regional, tratados comerciales.

\section{KEYWORDS}

Pyme Exporters, Regional Integration, Comercials Agrement.

\section{PLANTEAMIENTO DEL PROBLEMA}

¿Las Pymes exportadoras de Bogotá conocen las políticas otorgadas por el acuerdo marco de integración regional de la Unión de Naciones Suramericanas UNASUR? ¿Estas políticas son articuladas con la Alcaldía Mayor de Bogotá?

La metodología usada en este trabajo está estrechamente vinculada con la investigación documental y descriptiva. El documento se apoya en fuentes de carácter documental sobre la UNASUR, la integración regional y las políticas oficiales dentro del tratado. Igualmente generará una revisión bibliográfica que profundiza y dirige la investigación al análisis de la integración regional y las políticas para las Pymes exportadoras en la subregión, haciendo énfasis en el caso bogotano. Se incluyen también análisis existentes en fuentes secundarias oficiales y la sinergia entre las políticas enmarcadas en el acuerdo y las de carácter local establecidas actualmente por la Alcaldía Mayor de Bogotá teniendo presente la dinámica del sistema internacional y la política exterior. 


\section{INTRODUCCIÓN}

La integración regional es un proceso de cooperación que implica estrategias de negociación dinámica entre países con atributos nacionales y geográficos coincidentes. Países como los de nuestra región aprovechan las ventajas y beneficios de esta cercanía geográfica.

La integración industrialy productiva, con especial atención en laspequeñas y medianas empresas, las cooperativas, las redes $y$ otras formas de organización productiva.

La profesora Mora (2008) sostiene:

(...) que los procesos de negociación local y regional deben tenerse más en cuenta por las instituciones y los acuerdos, de manera tal que la integración local y regional sea un punto de partida para todo proceso negociador eficaz y eficiente, enmarcado en políticas que impliquen la dinamización del comercio y la organización y ejecución de una buena política exterior, enfocada en la integración como herramienta para los países de la subregión y aplicable especialmente hacia la UNASUR.

La página oficial de la UNASUR en su artículo 1 del Tratado constitutivo (2009), sostiene:

(...) La Unión de Naciones Suramericanas tiene como objetivo construir, de manera participativa y consensuada, un espacio de integración y unión en lo cultural, social, económico y político entre sus pueblos,

\section{RESEÑAS DE AUTORES}

Ángela Julieta Mora Ramírez

Politécnico Grancolombiano

(amoraram@poligran.edu.co)

(julietamora22@hotmail.com)

Economista, especialista en Integración en el Sistema Internacional. Cuenta con más de diez años de experiencia docente investigativa y escribe para publicaciones nacionales e internacionales. Es par de artículos en revistas indexadas y de divulgación. En la actualidad es docente investigadora de tiempo completo de la Institución Universitaria Politécnico Grancolombiano y dirige el Centro de Investigación Colombia Regional y Empresarial. otorgando prioridad al diálogo político, las políticas sociales, la educación, la energía, la infraestructura, el financiamiento y el medio ambiente, entre otros, con miras a eliminar la desigualdad socioeconómica, lograr la inclusión social y la participación ciudadana, fortalecer la democracia y reducir las asimetrías en el marco del fortalecimiento de la soberanía e independencia de los Estados.

Uno de los objetivos específicos del tratado constitutivo se encuentra en el artículo 3 de la UNASUR (2009).

m) la integración industrial y productiva, con especial atención en las pequeñas y medianas empresas, las cooperativas, las redes y otras formas de organización productiva.

Por lo anterior es necesario analizar las políticas de fomento y el papel de las Pymes en la subregión, haciendo hincapié en las Pymes exportadoras en Colombia que ofrecen un escenario de estudio por su aporte dinámico a la economía subregional.

Desde lo subregional a lo local, limitando el análisis a la ciudad de Bogotá, se busca establecer cómo las políticas regionales de la UNASUR y las políticas de la ciudad operan armónicamente en beneficio de las Pymes exportadoras de la región.

Este artículo hace parte de una investigación realizada por la autora en la Institución Universitaria Politécnico Grancolombiano. La metodología usada consta de dos partes: una inicial es la recolección documental descriptiva del proceso de integración desde la perspectiva de las Pymes exportadoras de la región limitando el estudio a Colombia. En la segunda, se realizara una verificación 
cuantitativa que servirá de herramienta de verificación y clasificación de las Pymes exportadoras en el país y su aporte al PIB. Aquí se enfatiza en la actual estrategia y dinámica internacional, especialmente para la ciudad de Bogotá.

\section{LA UNASUR Y SUS POLITICAS PARA LAS PYMES EXPORTADORAS}

Vachino Juan Mario (1981) supone la integración regional como proceso dinámico de generalización y aprovechamiento de las oportunidades potenciales de política exterior y la cercanía geográfica negociadora. En este sentido afirma:

(...) El INTAL define la integración como "un proceso de interdependencia estructural creciente que resulta de un esquema de interacción programada". Para esta definición, la medida del éxito efectivo de la integración estará dado justamente, por las interdependencias que logre establecer. Para el autor esta definición es "observable en algunos aspectos". En efecto, considera que entre una economía industrial y otra agropecuaria pueden establecerse estrechos lazos de interdependencia sin que exista integración, por falta de esa solidaridad que supone lazos tanto en la prosperidad como en la adversidad. De aquí concluye que, si bien puede haber interdependencia sin integración, no puede haber integración sin solidaridad.

La integración regional establece un proceso de cooperación teniendo presente la cercanía geográfica y algunas características comunes, como la idiosincrasia y la historia económica. De acuerdo con esto, la integración regional se establece con criterios geopolíticos claros.

Para el Centro de Investigación de Relaciones Internacionales y Desarrollo de Barcelona (2008):
(...) Desde los primeros años de la década de los noventa, distintos estados latinoamericanos han optado por las estrategias del llamado nuevo regionalismo para mejorar su inserción en el sistema internacional, y responder a distintos desafíos económicos, sociales, políticos y de seguridad planteados o agudizados por las dinámicas de la globalización. Quince años más tarde, el consenso existente sobre la validez de estas estrategias y la relevancia de la integración regional parece seguir vigente, si se toma como indicador la retórica gubernamental y el lanzamiento de iniciativas de amplio alcance como la Comunidad Sudamericana de Naciones (CSN), creada en 2005, y que en 2007 fue rebautizada como Unión de Naciones Sudamericanas (UNASUR). Sin embargo, esa retórica coexiste con un período de estancamiento o crisis de la integración regional, que es muy visible en Mercosur y en la Comunidad Andina de Naciones (CAN).

Para el Banco Interamericano de Desarrollo (BID), Fernando Quevedo y Luiz Villela (2003) dicen:

(...) Estos acercamientos regionales y generales de cooperación y nuevas estrategias llevaron a más estudios y análisis sobre estos temas. Por ejemplo, según el BID en respuesta a los desafíos que enfrenta América Latina y el Caribe, los diversos compromisos establecidos en cumbres recientes, incluyendo los Objetivos de Desarrollo del Milenio y la necesidad de asegurar la efectividad en el desarrollo, el BID ha elaborado un renovado marco estratégico. Este se inserta en el contexto de la estrategia Institucional y los mandatos de la más reciente reposición de recursos. El crecimiento económico sustentable y la reducción de la pobreza y promoción de la equidad social han sido definidos como los dos objetivos fundamentales de 
la institución. Éstos se atienden por medio de actividades en cuatro áreas prioritarias en las cuales el Banco tiene una ventaja comparativa: la modernización del Estado, la competitividad, el desarrollo social, y la integración regional.

Las políticas se especifican para los países andinos debido a la organización paulatina en la que se encuentra

la Unión de

Naciones Suramericanas.

Esta dinámica vista desde la integración regional supone una estrategia en la búsqueda del desarrollo de los países de la región, por vía de la negociación, especialmente en el actual escenario de interdependencia e incremento de las ventajas competitivas sobre las estrategias centralizadas.

La UNASUR y su propuesta de negociación vía integración regional tiene políticas específicas para las Pymes, como lo afirma la página oficial de la CAN (2008):

(...) El desarrollo y promoción de la Pyme requiere la adopción de instrumentos económicos y legales que provean un entorno favorable, y que faciliten procesos de diálogo y consulta entre los sectores privado y público involucrados en su desempeño. De ahí que se hace necesaria la creación del Estatuto Andino de la Pyme, un instrumento que establecerá los parámetros para la elaboración de políticas de fomento y desarrollo de la Pyme en los países andinos. Los objetivos que se persiguen con la creación del Estatuto Andino de la Pyme son: a) Identificar los temas prioritarios a desarrollar para favorecer el crecimiento y mejorar los niveles de competitividad y complementariedad de la Pyme andina. b) Articular propuestas de políticas, programas e instrumentos de promoción y desarrollo de la Pyme, en áreas de interés común, que permitan el crecimiento sostenido y sostenible de los sectores productivos de bienes y servicios de los países miembros. c) Promover la participación de los actores públicos y privados involucrados en el tema de la Pyme, de manera de articular esfuerzos y concretar iniciativas. d) Promover la armonización de los criterios de clasificación de la Pyme de los países miembros para facilitar la implementación de políticas comunitarias.

Lo anterior acompañado de políticas estratégicas internas que aseguren la permanencia de las Pymes en los mercados de los países Andinos, enfocado desde la CAN hacia la UNASUR. Las políticas se especifican para los países andinos debido a la organización paulatina en la que se encuentra la Unión de Naciones Suramericanas.

Continúo citando la página oficial de la CAN (2009) en la UNASUR:

(...) El proceso de globalización somete a las economías andinas a un conjunto de retos, lo que obliga a una revisión de las estrategias de desarrollo nacional, con el fin de superar la dependencia de tecnologías generadas en otros contextos, con la consiguiente pérdida de mercados por parte de nuestras empresas, aun en los mercados tradicionales. Esta situación es producto de las diferencias en las capacidades de generación de conocimiento entre nuestros países y países desarrollados y su eventual transformación de innovaciones que contribuyan a elevar el valor agregado de los bienes y servicios producidos por nuestras empresas. En los actuales momentos, debido a los diversos y simultáneos procesos de negociaciones comerciales en marcha, en los que se aspira hacerlo favorablemente intentando obtener mayores ventajas posibles frente a terceros, las pequeñas y medianas empresas corren el peligro de ser severamente afectadas por 
falta de capacidades competitivas, con los consiguientes problemas de destrucción del tramado productivo y la pérdida de empleo. La mejora de dichas capacidades competitivas exigen un proceso de mejoramiento continuo de la calidad de los productos desarrollados y ese mejoramiento tiene que ir acompañado de incorporación progresiva de innovaciones, tanto a nivel de los productos como de procesos. Podemos por lo tanto aseverar, que un desarrollo económico sostenido está fuertemente condicionado por la capacidad de incorporar conocimientos e innovación tecnológica a los sistemas productivos, como factor estratégico de competitividad.

Estos programas estratégicos del acuerdo de negociación subregional apoyan a las Pymes y establecen un enfoque más dinámico y estructural que genera mayor productividad y competitividad internacional. De la misma manera, establecen criterios base donde el desarrollo interno vía productiva es esencial en la consecución de una buena política de apoyo a la Pyme.

Las Pymes son parte esencial del desarrollo productivo y la CAN incluye propuestas que apoyen estas empresas y logren niveles aceptables de incorporación de su productividad.

Como cita la página oficial de la CAN (2009):

(...) Dado que las exportaciones de la región están constituidas fundamentalmente por productos primarios, con poco valor agregado y sometidos a los vaivenes de la demanda y a la erraticidad de los precios, debe hacerse un esfuerzo para darle un valor agregado, basado en la incorporación de innovaciones, mejoramiento de la calidad y mayor eficiencia en la producción y comercialización de dichos productos y servicios. Además, lograr y mantener competitividad requiere la integración real de las capacidades tecnológicas como país y como empresas, objetivo al cual deberá propender cualquier esfuerzo en ese sentido. Es importante entender que hay una relación indisoluble entre innovación y competitividad, para lo cual hay que hacer un esfuerzo que ayude a la adopción de políticas y toma de acciones que incrementen el desarrollo tecnológico de la región en función de la competitividad. Se reconoce que el "conocimiento" por sí mismo no transforma las economías o la sociedad, sino que puede hacerlo en el marco de sistemas nacionales o regionales de ciencia, tecnología e innovación, que posibiliten su incorporación al sector productor de bienes y servicios.

Todas estas iniciativas requieren dinero y movilización de capitales, según la página oficial de la CAN (2009):

(...) Una de las limitaciones que afecta el desempeño de las Pymes andinas es su dificultad para acceder a un crédito oportuno y barato, debido fundamentalmente a su debilidad en las garantías de respaldo y su alto índice de riesgo. Frente a esta situación, se ha acordado la creación de un Sistema Andino de Garantías para las Pymes, mecanismo comunitario que permitirá articular y fortalecer los esfuerzos que en materia de garantías vienen haciendo los países andinos.

El Sistema Andino de Garantía coadyuvará en el establecimiento de un mecanismo andino de reafianzamiento de garantía para liberar recursos de capital a nivel nacional con objeto de apalancar el otorgamiento de nuevas garantías, con los consiguientes beneficios para la Pyme de la región.
Se reconoce que el "conocimiento" por símismo no transforma las economías o la sociedad, sino que puede hacerlo en el marco de sistemas nacionales o regionales de ciencia, tecnología e innovación, que posibiliten su incorporación al sector productor de bienes $y$ servicios. 
La CAF no sólo emplea recursos propios, sino que aprovecha su poder de convocatoria para atraer recursos provenientes de otros inversionistas, bancos comerciales, bancos de desarrollo e instituciones públicas y privadas, tanto de la región como de otras partes del mundo.
Por otro lado, la principal fuente de financiamiento multilateral en la región es la Corporación Andina de Fomento (CAF), que tiene su sede principal en Venezuela, inició actividades en 1970 y en la actualidad cuenta con diecisiete países accionistas. Partiendo del hecho de que la CAF “...está comprometida con el desarrollo sostenible y la integración regional, pilares de su misión" tal como se lee en su página oficial, cabría preguntarse qué políticas tiene esta institución para las Pymes pertenecientes a los países miembros. En la página oficial de la UNASUR se encuentra:

(...) El apoyo y fortalecimiento de la Pyme es un reto fundamental dentro del plan de acción de la CAF. Por esta razón, además de las operaciones con instituciones micro financieras, la CAF desarrolla novedosos mecanismos para financiar a la pequeña $y$ mediana empresa.

La CAF no sólo emplea recursos propios, sino que aprovecha su poder de con- vocatoria para atraer recursos provenientes de otros inversionistas, bancos comerciales, bancos de desarrollo e instituciones públicas y privadas, tanto de la región como de otras partes del mundo.

Así se observa el empleo de recursos tanto públicos como privados por parte de la CAF. Debe resaltarse el hecho de que el correcto desarrollo de las Pymes en la UNASUR favorece y, por lo tanto, debería concernir tanto a los gobiernos como a la empresa privada no sólo entendido en el marco de la cooperación sino también, visto como oportunidad de negocio.

Contando con una idea más clara sobre algunas políticas de la UNASUR para las Pyme se pasará ahora a ver cómo funcionan éstas en Colombia y qué políticas tiene el país para favorecerlas. Primero será pertinente entender la participación del país en la UNASUR a través de su PIB y exportaciones.

\section{UNASUR (UNIÓN DE NACIONES SURAMERICANAS)}

\begin{tabular}{|c|c|c|c|c|c|c|c|}
\hline & \multicolumn{2}{|c|}{ EXPORTACIONES } & & & \multicolumn{2}{|c|}{ PIB } \\
\hline & & ANO 2007 & $\%$ & & & ANO 2007 & $\%$ \\
\hline MERCOSUR & ARGENTINA & 55.932 & $12 \%$ & MERCOSUR & ARGENTINA & 260.140 & $11 \%$ \\
\hline & BRASIL & 160.619 & $35 \%$ & & BRASIL & 1.313 .590 & $56 \%$ \\
\hline & PARAGUAY & 3.374 & $1 \%$ & & PARAGUAY & 10.870 & $0 \%$ \\
\hline & URUGUAY & 4.496 & $1 \%$ & & URUGUAY & 22.951 & $1 \%$ \\
\hline CAN & BOLIVIA & 4.814 & $1 \%$ & CAN & BOLIVIA & 13.192 & $1 \%$ \\
\hline & COLOMBIA & 29.987 & $7 \%$ & & COLOMBIA & 171.607 & $7 \%$ \\
\hline & ECUADOR & 13.649 & $3 \%$ & & ECUADOR & 44.184 & $1 \%$ \\
\hline & PERU & 27.857 & $6 \%$ & & PERU & 109.069 & $5 \%$ \\
\hline IND & CHILE & 67.644 & $15 \%$ & IND & CHILE & 163.792 & $7 \%$ \\
\hline & VENEZUELA & 83.130 & $18 \%$ & & VENEZUELA & 236.390 & $10 \%$ \\
\hline & GUYANA & 681 & $1 \%$ & & GUYANA & 934 & $0 \%$ \\
\hline & SURINAM & 960 & $1 \%$ & & SURINAM & 2.234 & $1 \%$ \\
\hline TOTAL & JNASUR & 453.143 & $100 \%$ & & & 2.348 .953 & $100 \%$ \\
\hline
\end{tabular}

Fuente: datos seleccionados Semillero Juan Carlos Piñeros basado de Secretaría General, Comunidad Andina. Página oficial www.comunidadandina.org (2009). Datos de 2008. 
EXPORTACIONES UNASUR

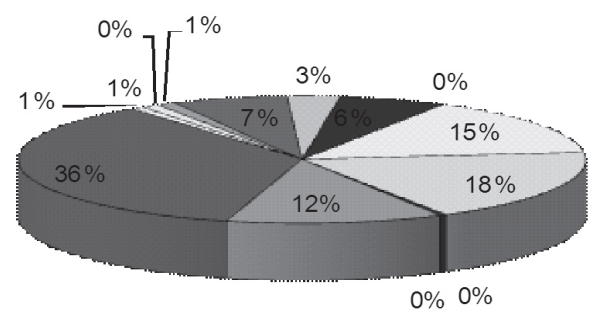

CMERCOSUR ARGENTINA 12\%

-MERCOSURBRASIL $36 \%$

ᄃMERCOSUR PARAGUAY $1 \%$

CMERCOSUR URUGUAY 0\%

ᄃCAN BOLIVIA $1 \%$

-CAN COLOMBIA 7\%

CCAN ECUADOR 3\%

- CAN PERU $\quad 6 \%$

ㄷINDCHILE $\quad 15 \%$

CIND VENEZUELA $18 \%$

- IND GUYANA $0 \%$

- IND SURINAM $\quad 0 \%$

Fuente: Secretaría General, Comunidad Andina. Página oficial www.comunidadandina.org (2009). Datos de 2008.

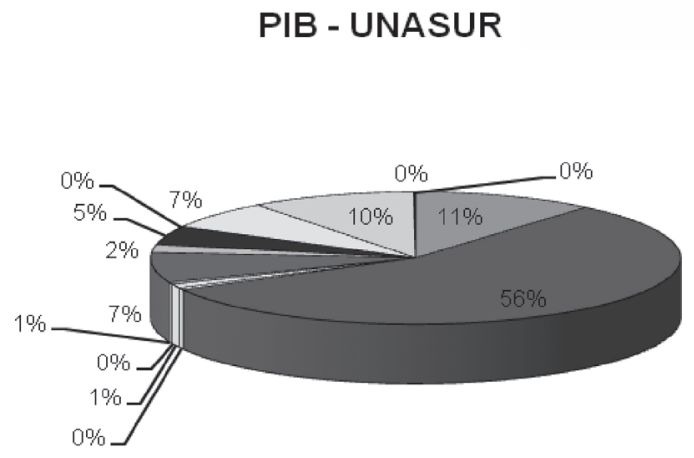

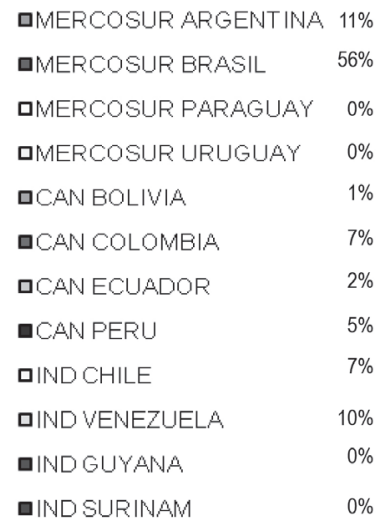

Fuente: Secretaría General, Comunidad Andina. Página oficial www.comunidadandina.org (2009). Datos de 2008.

Se observa así cómo Colombia figura en el quinto lugar en participación de exportaciones y su PIB es con el de Chile, el cuarto más grande de la región.

Esto es representativo teniendo presente que la producción interna del país depende de las grandes empresas y una cuantificación adicional dirigida a las empresas exportadoras Pymes.

\subsection{LAS PYMES EN COLOMBIA}

Hay que partir del hecho de que la actual tendencia mundial hacia la globalización no puede ser soslayado. Indiferentemente de las creencias personales o colectivas, la apertura de los mercados va a ser tarde o temprano una realidad hacia la cual, con retrocesos y demoras, ya están apuntando las naciones. Más que enfrascarse en discusiones teóricas sobre las bondades o inconvenientes de un comercio internacional libre de barreras hay que ser pragmáticos y afrontar de la mejor manera posible las condiciones que se presentan. Es precisamente en estas condiciones de integración progresiva que el fortalecimiento de las Mipymes en Colombia aparece como un requerimiento indiscutible para el éxito del país en procesos de integración y podría estar enfocado a potencializar el desarrollo 
endógeno, pensado desde la internacionalización de las economías.

En Colombia, la definición legal más reciente de las Pymes, o más exactamente de las Mipymes (siglas de micro, pequeña y mediana empresa), pude ser encontrada en la Ley No. 905 (2 de agosto de 2004):

(...) Por medio de la cual se modifica la Ley 590 de 2000 sobre promoción del desarrollo de la micro, pequeña y mediana empresa colombiana y se dictan otras disposiciones.

Donde las Mipymes se delimitan a toda unidad de explotación económica realizada por persona natural o jurídica. En el cuadro que se muestra a continuación hay un resumen sobre la clasificación de Mipymes en Colombia.

\begin{tabular}{|lcc|}
\hline \multicolumn{1}{|c}{ Tipo } & Cantidad trabajadores & Total activos \\
\hline Mediana empresa & Entre 51 y 200 & Entre 5001 a 30.000 SMMLV \\
\hline Pequeña empresa & Entre 11 y 50 & Entre 501 y 5000 SMMLV \\
\hline Microempresa & No superior a 10 & Hasta 500 SMMLV (excluida vivienda) \\
\hline
\end{tabular}

Fuente: www.desarrolloeconomico.gov.co(2009). Datos de 2008.

La empresa debe cumplir conjuntamente las dos características señaladas en la tabla mostrada arriba para entrar en uno de los tipos determinados (micro, pequeña o mediana empresa).

Para entender la importancia de las Mipymes en Colombia se deben tener presente los siguientes datos:

En la página $w w w . b u s i n e s s c o l . c o m$ (2009) se ve sobre las Mipymes:

(...) Representan el 96,4\% de los establecimientos, aproximadamente el 63\% del empleo; el $45 \%$ de la producción manufacturera, el $40 \%$ de los salarios y el $37 \%$ del valor agregado. Son más de 650.000 empresarios cotizando en el sistema de seguridad social. Además, es bueno resaltar que aproximadamente el $85 \%$ de las empresas colombianas son Mipymes pero que la mayoría de las ventas son hechas por la gran empresa.

Tal como se observa en el siguiente cuadro tomado de la Revista Dinero (2009):

\begin{tabular}{|c|c|c|c|c|c|c|c|c|c|c|c|}
\hline \multirow[b]{2}{*}{ TIPO DE EMPRESA } & \multirow{2}{*}{$\begin{array}{l}\text { CATEGORÍA DE CLASFFCACIÓN DE } \\
\text { LAS EMPRESAS POR SU NIVEL DE } \\
\text { VENTAS (\$MMLLONES) }\end{array}$} & \multicolumn{2}{|c|}{ EMPRESAS } & \multicolumn{3}{|c|}{ VENTAS EN 2007} & \multicolumn{5}{|c|}{ EXPORTACIONES EN 2007} \\
\hline & & NO. & $\begin{array}{c}\text { PARTICIPACl } \\
\text { ÓN\% }\end{array}$ & \$BBLLONES & $\begin{array}{l}\text { VARIACIÓN } \\
\text { ANUAL\% }\end{array}$ & $\begin{array}{c}\text { PARTILCPAC } \\
\text { IÓN\% }\end{array}$ & $\begin{array}{c}\text { NO. } \\
\text { EMPRESAS }\end{array}$ & $\begin{array}{c}\text { PARTICPAC } \\
\text { ION \% }\end{array}$ & USȘ MILLONES & $\begin{array}{l}\text { VARIACIÓN } \\
\text { ANUAL\% }\end{array}$ & $\begin{array}{c}\text { PARTICIPACl } \\
\text { ON \% }\end{array}$ \\
\hline GRANDE (D) & MAYORA $\$ 500.000$ & 129 & 0,5 & 222,5 & 16,0 & 43,7 & 81 & 2,2 & 28598 & 5,8 & 72,8 \\
\hline $\operatorname{GRANDE}(C)$ & ENTRE $\$ 100.000$ Y $\$ 500.000$ & 553 & 2,0 & 110,5 & 20,5 & 21,7 & 295 & 8,2 & 5733 & 12,5 & 14,6 \\
\hline GRANDE (B) & ENTRE $\$ 30.000$ Y $\$ 100.000$ & 1517 & 5,5 & 80,2 & 22,4 & 15,7 & 508 & 14,1 & 2785 & 11,6 & 7,1 \\
\hline GRANDE(A) & ENTRE $\$ 15.000$ Y $\$ 30.000$ & 1711 & 6,2 & 36,3 & 24,4 & 7,1 & 456 & 12,6 & 868 & 9,2 & 2,2 \\
\hline MEDIANA & ENTRE $\$ 5.000 Y \$ 15.000$ & 4186 & 15,2 & 36,8 & 19,6 & 7,2 & 1030 & 28,5 & 974 & 5,8 & 2,5 \\
\hline PEQUEÑA & ENTRE $\$ 1.000$ Y $\$ 5.000$ & 7977 & 29,0 & 19,4 & 10,8 & 3,8 & 978 & 27,1 & 277 & $-15,3$ & 0,7 \\
\hline MICRO & MENORA $\$ 1.000$ & 11468 & 41,6 & 3,7 & $-25,8$ & 0,7 & 260 & 7,2 & 22 & $-44,9$ & 0,1 \\
\hline TOTAL & & 27541 & 100,0 & 509,3 & 18,0 & 100,0 & 3608 & 100,0 & 39257 & 7,0 & 100,0 \\
\hline
\end{tabular}

Fuente: Revista Dinero 
Por otro lado, mientras el aporte al PIB del país por parte de las empresas grandes corresponde al 55,0\% y el de las Pymes tan sólo al 38,7\%, el porcentaje de empleo contrastantemente es del $25 \%$ y el $70 \%$ respectivamente.

Después de tener una idea global de lo que son las Mipymes, y algunos datos estadísticos relevantes de las mismas en Colombia, se entenderá la importancia de estas empresas en el país en términos generales económicos y específicamente el impacto en los procesos de integración regionales, sobre todo de aquellas Mipymes que van a contribuir favorablemente a la balanza comercial. Esto significa: las Mipymes exportadoras. Será conveniente entonces analizar qué incentivos tiene el Gobierno nacional y particularmente la Alcaldía Mayor de Bogotá para estas empresas. Antes de hablar sobre los incentivos existentes para las Mipymes exportadores se hará un resumen de dichos incentivos para las Mipymes en general.

\section{INCENTIVOS A LAS MIPYMES}

El Gobierno de Colombia consciente de la importancia de la creación, permanencia y consolidación de la micro, pequeña y mediana empresa ha creado normas, políticas, ayudas, planes, programas y proyectos para lograr tales objetivos en el plano nacional, al igual que en el plano distrital en Bogotá.

La Alcaldía Mayor de Bogotá, a través de la Secretaría Distrital de Desarrollo Económico y conociendo la falta de divulgación de información pertinente a la que se ven enfrentados muchos empresarios de la ciudad, elaboró dos documentos sobre incentivos, privilegios, apoyos y estímulos para las Mipymes, por parte tanto del
Gobierno nacional como del distrital. El primero de estos documentos se llama "Incentivos para las Mipymes industriales, agroindustriales, comerciales y de servicios" y el segundo "Incentivos para las MIPYMES exportadoras” que básicamente muestra las partes que competen a la exportación del primer documento. Los dos documentos del año 2008 se pueden encontrar en la página de internet http:// www.desarrolloeconomico.gov.co y son clave para el desarrollo de esta investigación.

El texto "Incentivos para las Mipymes industriales, agroindustriales, comerciales y de servicios" afirma:

(...) Para facilitar la comprensión de los temas y dado su mayor número, en la primera sección se hará referencia a los incentivos relacionados con la financiación de las Mipymes, tanto directos como indirectos; luego, se presentarán los incentivos indirectos, en el siguiente orden: servicios, privilegios de mercado, fiscales y de reglamentación.

A lo largo del texto se dan a conocer las entidades encargadas de cada uno de los incentivos que se mencionan en esta cita, así como la definición, el marco legal, el objeto, los beneficiarios y en general todos aquellos aspectos importantes sobre éstos.

\section{INCENTIVOS A LAS MIPYMES EXPORTADORAS}

Teniendo ya una idea general de los incentivos existentes para las Mipymes se centrará ahora la atención en los incentivos específicos para Mipymes exportadoras. Estas empresas tienen los mismos incentivos que los mencionados bajo el subtítulo anterior. A continuación se mencionan aquellos
Se entenderá la importancia de estas empresas en el país en términos generales económicos y específicamente el impacto en los procesos de integración regionales, sobre todo de aquellas Mipymes que van a contribuir favorablemente a la balanza comercial. Esto significa: las Mipymes exportadoras. Será conveniente entonces analizar qué incentivos tiene el Gobierno nacional y particularmente la Alcaldía Mayor de Bogotá para estas empresas. 
La zona franca

es el área

geográfica

delimitada

dentro del

territorio

nacional,

en donde se

desarrollan

actividades

industriales

debienesy

de servicios,

o actividades

comerciales,

bajo una

normatividad

especialen

materia

tributaria,

aduaneray

de comercio

exterior. beneficios que son de mayor ayuda a la actividad exportadora de las Mipymes.

Con seguridad el incentivo más conocido y usado por los empresarios involucrados en el comercio internacional debido a sus grandes ventajas es el Plan Vallejo, que inicia en 1959. Sobre qué es el Plan Vallejo se lee en el documento "Incentivo para las Mipymes exportadoras" lo siguiente:

(...) Es el régimen que permite a personas naturales o jurídicas, que tengan el carácter de empresarios, productores, exportadores, comercializadores o entidades sin ánimo de lucro, importar temporalmente al territorio aduanero colombiano, con exención total o parcial de derechos de aduana e impuestos: insumos, materias primas, bienes intermedios o bienes de capital y repuestos que se utilicen en la producción de bienes de exportación o que se destinen a la prestación de servicios directamente vinculados a la producción o exportación de estos bienes.

El Gobierno entendiendo la importancia de las exportaciones para el país, favorece aquellas importaciones que contribuyan a las personas jurídicas o naturales con el propósito de que pongan sus productos, bien sean bienes o servicios, en el exterior eximiéndolos de impuestos. Este plan puede ser usado por Mipymes al igual que por empresas grandes.

Continuando con el documento "Incentivo para las Mipymes exportadoras" del año 2008, otro aspecto a destacar es el relacionado con las zonas francas y su uso. Se lee allí:

(...) La zona franca es el área geográfica delimitada dentro del territorio nacional, en donde se desarrollan actividades indus- triales de bienes y de servicios, o actividades comerciales, bajo una normatividad especial en materia tributaria, aduanera y de comercio exterior. Las mercancías ingresadas en estas zonas se consideran fuera del territorio aduanero nacional para efectos de los impuestos a las importaciones y a las exportaciones.

La finalidad de las zonas francas se describe en el documento así:

1. Ser instrumento para la creación de empleo y para la captación de nuevas inversiones de capital.

2. Ser un polo de desarrollo que promueva la competitividad en las regiones donde se establezca.

3. Desarrollar procesos industriales altamente productivos y competitivos, bajo los conceptos de seguridad, transparencia, tecnología, producción limpia, y buenas prácticas empresariales.

4. Promover la generación de economías de escala.

5. Simplificar los procedimientos del comercio de bienes y servicios, para facilitar su venta.

La correcta utilización de las zonas francas es una de las ventajas competitivas que tiene cualquier país en el mundo. El aprovechamiento de éstas por parte de las Mipymes en Colombia es de vital importancia para los procesos de integración que favorezcan la competitividad del país.

Estos dos incentivos: Plan Vallejo y zonas francas, tuvieron mayor impacto para las Mipymes exportadoras. Sin embargo, existen otros como: sociedades comercializadoras internacionales, usuario aduanero permanente, usuario altamente exportador (ALTEX), depósitos privados 
para procesamiento industrial, programas especiales de exportación, etcétera.

\section{LAS MIPYMES EXPORTADORAS:PERCEPCIONES DE LOS EMPRESARIOS}

En la "Encuesta de comportamiento y opinión de la pequeña y mediana industria de Colombia" realizada por la Asociación Nacional de Micro, Pequeñas y Medianas Empresas (ACOPI) y la Fundación Konrad Adenauer de Alemania (KAS) con respecto al primer trimestre del 2008 se encuentran algunos datos elocuentes sobre la percepción del los empresarios concernientes al comportamiento de las Mipymes.

(...) Cabe señalar el hecho de que la encuesta fue realizada en una muestra de 245 empresas de una población total de 9.186 (el 2,67\%).

Entre la información relevante para el desarrollo de este estudio de la encuesta mencionada, se encontró la ratificación de la conocida concentración de las Mipymes en el mercado local: el 86,7\% de los encuestados parecen no tener ninguna producción dirigida al mercado externo.

Por otro lado, el marco de integración regional por medio de los acuerdos internacionales parece no tener mayor impacto entre los empresarios. Sobre la incidencia de éstos en el comportamiento de las exportaciones el documento dice :

(...) Para el 6,1\% de los empresarios que respondieron esta sección, los acuerdos internacionales que se promueven están incidiendo favorablemente, comparativamente con igual período de 2.007; mientras que en el semestre inmediatamente anterior el $14,8 \%$ de los empresarios se pronunciaron en ese sentido.
Esto es importante en la medida en que la ciudad masifique y visibilice sus políticas en las Pymes exportadoras. Éstas puedan conocer más sobre los tratados y acuerdos de negociación y pueden generar nuevas propuestas y apoyo para lograr acceder al mercado subregional de manera dinámica y amplia.

La articulación existente debe abarcar todo el proceso de integración y pasarlo de lo general a lo particular y de lo nacional a lo local, por medio de iniciativas puntuales de cooperación y desarrollo productivo de las Pymes exportadoras.

\section{DISCUSIÓN}

La integración regional en Latinoamérica surge como una oportunidad para enfrentar de manera adecuada los diferentes retos que se presentan en el actual mundo global. Tal integración debe ser entendida desde el marco de cooperación internacional favorecida por la cercanía geográfica existente y las similitudes culturales del territorio. La UNASUR es la iniciativa latinoamericana de integración para insertar de la mejor manera a sus países miembros dentro del sistema internacional.

Teniendo presente la importancia de estos procesos de integración, ahora conviene analizar a los participantes de los mismos. Ellos son los que finalmente llevarán a buen término lo planteado desde los dirigentes regionales. Cabe así investigar el papel que desempeñan las Pymes como agentes de gran impacto dentro de la UNASUR.

Se encontró que la UNASUR, consciente de la importancia de las Pymes, tiene políticas para fomentar su creación, desarrollo, consolidación y mejoramiento. Tales políticas en el plano supranacional se consolidan
La UNASUR es la iniciativa latinoamericana de integración para insertar de la mejor manera a sus países miembros dentro del sistema internacional. 
dentro de cada país siguiendo los lineamientos propios de los Estados miembros.

Particularmente en Colombia se tienen incentivos tanto directos como indirectos para la consolidación de las Mipymes. Dentro de los procesos de integración las Mipymes que van a tener mayor impacto son aquellas que por su actividad exportadora-importadora van a afectar su balanza comercial. Particularmente convienen al país las empresas que ayudan favorablemente tal balanza es decir, las exportadoras. Es pertinente entonces conocer y dar a conocer los incentivos a las Mipymes exportadoras.

Específicamente en Bogotá, las Mipymes exportadores tienen a su disposición un documento de vital importancia que las puede guiar a la hora de hacer uso de los incentivos dados por el Gobierno. Tal documento es el "Incentivo para las Mipymes exportadoras" elaborado por la Secretaria Distrital de Desarrollo Económico de la Alcaldía Mayor de Bogotá este año y que puede ser encontrado en la página de internet http://www.desarrolloeconomico.gov.co. En este documento se da información detallada de incentivos tales como el Plan Vallejo o de aquellos relacionados con el uso de las zonas francas.

Se puede decir que tanto la Alcaldía Mayor de Bogotá como el Gobierno nacional de Colombia tienen políticas claras de favorecimiento a las Mipymes y que tales políticas pueden ser conocidas por los interesados a través de medios de comunicación como Internet. Esto también es aplicable a las políticas elaboradas por la UNASUR. Sin embargo, teniendo presente la percepción de los empresarios y el impacto de tales esfuerzos por dar a conocer la información, parece ser aún insuficiente y susceptible de ser ampliado y mejorado.

Conviene llegar al empresario de la micro, pequeña y mediana empresa de manera más contundente, no focalizándose en alguno de ellos sino más bien, teniendo presente a los tres. Programas de mayor impacto se vuelven así necesarios para que las buenas intenciones de las políticas planteadas en la UNASUR y dentro del país no lleguen a ser conocidas sólo por unos pocos empresarios.

La estructura de los acuerdos comerciales permite a las Pymes exportadoras involucrarse en el mercado internacional, pero la sincronía entre lo subregional, lo nacional y local debe establecer una mayor imbricación, en cuanto que el papel del Estado y de las Pymes de la ciudad pueden generar nuevos enfoques internacionales, más aún sabiendo que las exportaciones de la subregión, desde las Pymes en Bogotá, representan un actor importante para la movilidad de capital de la nación.

El tema de las Pymes es de gran importancia y requiere estudios y análisis de profundidad. Este artículo es un apoyo a la investigación de tipo descriptivo y documental realizada en la Institución Universitaria Politécnico Grancolombiano con el apoyo del estudiante de semillero Juan Carlos Piñeros.

Este proyecto es parte de un trabajo de investigación aplicado, en el que los enfoques y el desarrollo de los temas son esenciales para la ciudad, el país y los tratados internacionales, todo con una base pedagógica e investigativa en función del crecimiento y desarrollo de la investigación en nuestra institución universitaria.

La articulación entre la integración desde la UNASUR, las Pymes y las políti- 
cas nacionales y locales debe generar una dinámica en la que se pueda establecer una sincronía entre procesos y se logre dinami- zar la estrategia regional con miras al desarrollo y crecimiento del sector de las Pymes y la estructura empresarial en general.

\section{BIBLIOGRAFÍA}

- Alcaldía Mayor de Bogotá. (2009). Secretaría Distrital de Desarrollo Económico. Dirección de Estudios Socioeconómicos y Regulatorios. Incentivo para las MIPYMES. Disponible en internet en: $h t t p: / / w w w$.desarrolloeconomico.gov.co. Consultado el 10 de Marzo de 2009.

- Asociación Nacional de Micro, Pequeñas y Medianas Empresas, (ACOPI). (2009). Encuesta de Comportamiento y Opinión de la Pequeña y Mediana Industria de Colombia. Informe de Resultados Segundo Semestre de 2008. Bogotá.

- Ballassa, Bella. (1961). The Theory of Economic Integration. Uteha: México 1964. Citado por la autora en investigación ZIF Colombia y Venezuela desde el funcionalismo. Material documental. Universidad Politécnico Gran Colombiano. Revista Panorama 2008. Bogotá. Colombia 2009.

- Centro de Investigación de Relaciones Internacionales y Desarrollo. Actividades del CIDOB. (2009).Seminario: La integración regional en América del Sur. Barcelona: Programa América Latina de la Fundación CIDOB. Disponible en: http://www.cidob.org/ es/actividades/america_latina/la_integracion_regional_en_america_del_sur. Recuperado 11 de febrero de 2009.

- Comunidad Andina de Naciones. CAN. (2008). Principales Indicadores de la Unión de Naciones Suramericanas 1998-2007. Obtenido el 10 de marzo de 2009, de $h t t p: / / w w w$. comunidadandina.gov.

- Fondo Monetario Internacional (FMI Consultado). (2008). La globalización: ¿Amenaza u oportunidad?. Obtenido el 9 de febrero de 2008, de http://laberinto.uma.es/index. php?option $=$ com_content $\&$ task $=$ view $\&$ id $=18 \&$ Itemid $=30$.

- Maillat, D. (1995). Territorial Dynamic, Innovative Milieus and Regional Policy. Entrepreneurship \& Regional Development, (7):157-165. Obtenido el 12 de febrero de 2009, de http://www.scielo.cl.

- Mora Ramírez, A. J. (2008). Zona de Integración Fronteriza Colombia- Venezuela desde el funcionalismo. En Revista Panorama: 35-54.

- Quevedo, Fernando y Luiz Villela. Banco Interamericano de Desarrollo. (BID). Integración regional, documento de estrategia. Obtenido el 12 de febrero de 2009, de http://74.125.47.132/search?q=cache:TCixxCCdyioJ:www.iadb.org/document.cfm\%3Fid $\% 3 \mathrm{D} 1448827+$ integracion + regional\&hl=es\&ct $=\mathrm{clnk} \& \mathrm{~cd}=5 \& \mathrm{gl}=$.

- Revista Dinero. (2008). La Pyme Evoluciona. Obtenido el 10 de marzo de 2009, de http:// www.dinero.com/wf_InfoArticulo.aspx?idArt $=48941$

- Sunkel, O. El desarrollo de América Latina ayer y hoy. CDC. [online]. Obtenido el 26 de febrero de 2009, de http://www.scielo.org.ve/scielo.php?script=sci_arttext\&pid=S101225082005000300007\&lng=es\&nrm=iso >. ISSN 1012-2508. Consultado 26 de febrero de 2009.

- undp. Citando por TICs. (2009). Disponible en: http://www.undp.org/spanish/.

- Vázquez-Barquero, A. Desarrollo endógeno y globalización. En EURE. Obtenido el 20 de febrero de 2009, de http://www.scielo.cl/scielo.php?pid=S025071612000007900003\&script =sci_arttext\&tlng=en\#img 01 . 